\title{
Nail Unit Schwannoma: An Important Consideration in the Differential Diagnosis of Soft Tissue Tumors Affecting the Nail Apparatus
}

\author{
Mohammed Dany ${ }^{a} \quad$ Andrew S. Fischer ${ }^{a}$ Susan Pei ${ }^{a}$ Temitayo Ogunleye ${ }^{a}$ \\ Rosalie Elenitsas ${ }^{a}$ Adam I. Rubin ${ }^{a, b, c}$

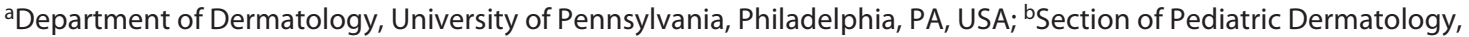 \\ Children's Hospital of Philadelphia, Philadelphia, PA, USA; 'Department of Pathology and Laboratory Medicine, \\ Children's Hospital of Philadelphia, Philadelphia, PA, USA
}

\section{Established Facts}

- Schwannomas are relatively uncommon tumors that present as solitary, soft, skin-colored dermal or subcutaneous papulo-nodules most commonly on the flexor part of extremities and head and neck areas.

\section{Novel Insights}

- Nail unit schwannoma is a rare tumor of the nail apparatus with only four prior reports in the literature.

- Nail unit schwannoma should be considered in the clinical differential diagnosis of subungual soft tissue tumors. Both dermatologists and dermatopathologists need to be aware of this entity for correct sampling, diagnosis, and patient counseling.

- Our review of the literature and analysis of the current case demonstrates that the average age of patients with nail unit schwannoma is 39 years, with the affected sites being the thumb, ring finger, third finger, and great toe. All reports performed an excisional biopsy except in our case where we performed superficial and deep shave biopsies with extraction of the tumor with forceps.

\section{Keywords}

Schwannoma - Neurilemmoma - Nail unit · Soft tissue tumor

\begin{abstract}
Schwannoma is a tumor of schwann cell proliferation which presents as a solitary, soft, skin-colored dermal or subcutaneous papulo-nodule most commonly on the flexor part of
\end{abstract}

extremities and head and neck areas. Here, we report a case of nail unit schwannoma, which is a rare tumor of the nail apparatus with only 4 other prior reports in the literature. This case illustrates the importance of including subungual schwannoma in the clinical differential diagnosis of subungual soft tissue tumors. We include a literature review which catalogs and summarizes the current knowledge regarding this unusual nail unit neoplasm.

(c) 2020 S. Karger AG, Basel

$\begin{aligned} & \text { karger@karger.com } \\ & \text { www.karger.com/sad }\end{aligned}$
Karger $\%$ 2020 S. Karger AG, Basel




\section{Introduction}

Schwannoma, also known as neurilemmoma, is a benign uncommon tumor of the nerve sheath composed entirely of schwann cells. The tumor is hypothesized to be caused by a proliferation of Schwann cells, secondary to mutations in the NF2 gene. The normal nerve fiber endings are pushed to the periphery of the tumor, and the proliferating cells remain encapsulated within the perineurium [1]. Clinically, schwannoma presents as a soft, skin-colored dermal or subcutaneous papulo-nodule [2]. It is most commonly found on the flexor part of extremities. The head and neck area is the second most common location [1]. They are usually painless but can become tender, especially when the tumor impinges on pain fibers. The vast majority of schwannomas are solitary and not associated with a syndrome; however, a small subset of patients with schwannomas have neurofibromatosis type 2 and have other features such as acoustic neuromas and meningiomas [3]. Another small subset of patients who have multiple schwannomas are referred to as having neurilemmomatosis. This is usually associated with germline mutations in SMARCB1, an actin-dependent regulator of chromatin [4]. Another rare variant of schwannoma presents clinically as a blue tumor called psammomatous melanotic schwannoma, which is found in patients with Carney complex [5].

\section{Case Report/Case Presentation}

A 24-year-old man presented with a 5-month history of a tender lesion affecting the nail unit of the left third finger. His other medical history was unremarkable. The patient described that the lesion affecting the nail unit was progressively painful and was increasing in size over time. On physical exam, the left third fingernail unit demonstrated erythema and swelling which involved the hyponychium, lateral nail fold, and nail bed (Fig. 1). He was also found to have nail pitting in several nail plates. A superficial shave biopsy was performed initially in the area of apparent swelling, revealing a deeper mass. A deeper shave biopsy was performed directly afterward and demonstrated a nodular tumor which was able to be extracted with forceps through the biopsy site. A few interrupted sutures were placed to close the defect and achieve hemostasis.

Histopathologically, the low-power view of the lesion demonstrated a well-circumscribed, encapsulated tumor composed of hypercelleular (Antoni A-type tissue) and hypocellular (Antoni Btype tissue) areas. The hypercellular areas were composed of cells with elongate and spindle nuclei embedded within an eosinophilic matrix (Fig. 2a). There were multiple areas where the nuclei were aligned in parallel rows separated by acellular zones, also known as Verocay bodies (Fig. 2b, c). Lesional mitotic figures were absent. The morphology of the cells and the presence of Verocay bodies

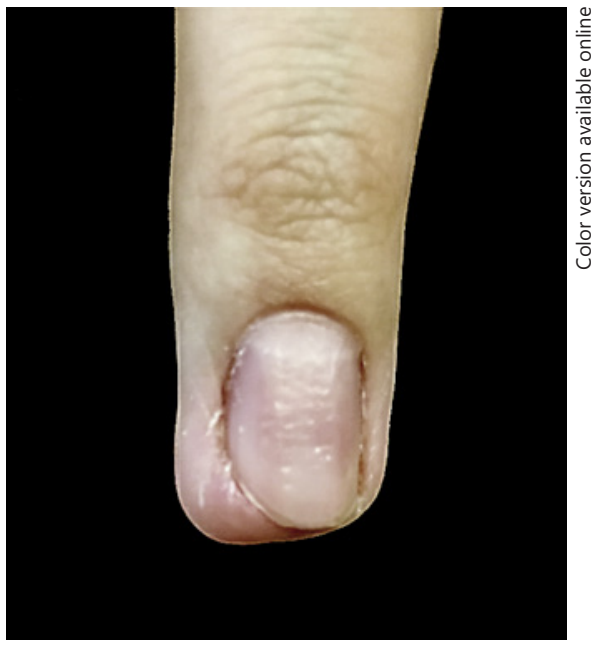

Fig. 1. Erythema and swelling involving the hyponychium, lateral nail fold, and nail bed on the left third fingernail.

are characteristic of a schwannoma. The patient was told to monitor for recurrence or persistence of symptoms.

\section{Discussion/Conclusion}

We describe a patient with a nail unit schwannoma that presented as an acquired soft tissue mass. Nail unit schwannomas are rare. Schwannomas, also known as neurilemmomas, usually present on the flexor parts of extremities or in the head and neck area. There are only four other reported subungual schwannomas in the literature (Table 1) [6-9]. The first reported case was in 2006 by Moon et al. [7] in a 49-year-old man on his right thumb for 8 years. The authors excised the tumor using a transungual approach, and postoperatively, the nail plate grew normally with no recurrence of the tumor at 12-month follow-up. The second case was reported in 2006 by Huntley et al. [8] in a 35-year-old woman who presented with a painless subungual lesion affecting the right thumb for 4 years. The authors performed an excisional biopsy after the nail was partly avulsed and confirmed the diagnosis of schwannoma histopathologically. The third case was reported in 2013 by Kulkarni et al. [6] in a 26-year-old man who presented with a painless tumor arising from the distal nail bed with bony erosion and fingertip destruction. The tumor had increased in size over 14 years with no history of trauma. The tumor was resected and reconstruction of the fingertip was performed with a V-Y flap. The diagnosis of schwannoma was confirmed histopathologically, and postoperatively, 


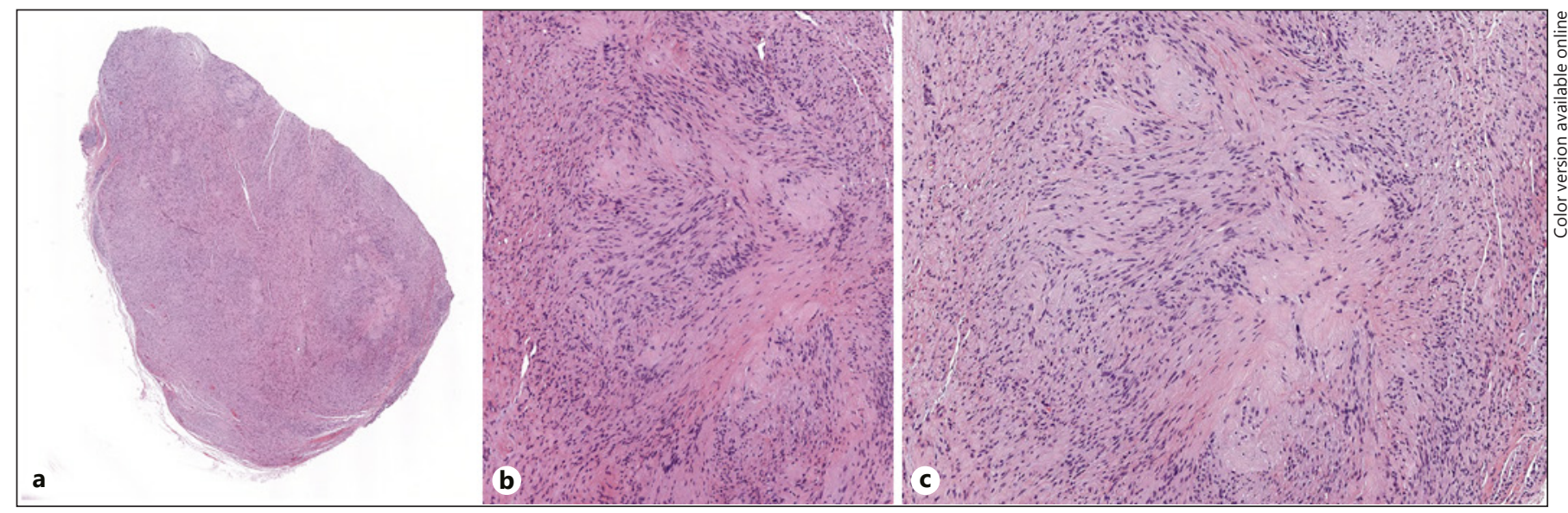

Fig. 2. a Scanning power magnification reveals a well-circumscribed, encapsulated tumor composed of hypercellular and hypocellular areas $(\mathrm{H} \& \mathrm{E}, \times 20)$. b Multiple areas with alignment of nuclei in parallel rows separated by acellular zones, also known as verocay bodies $(\mathrm{H} \& \mathrm{E}, \times 55)$. c Higher power showing a verocay body $(\mathrm{H} \& \mathrm{E}, \times 100)$.

Table 1. Reports of nail unit schwannoma

\begin{tabular}{lllllll}
\hline Authors & Year & Age, years & Gender & Location & Type of biopsy & Follow-up \\
\hline Moon et al. [7] & 2006 & 49 & Male & Right thumb & Excision after nail plate avulsion & No recurrence at 12 months \\
Huntley et al. [8] & 2006 & 35 & Female & Right thumb & Excision after partial nail plate avulsion & Unknown \\
Kulkarni et al. [6] & 2013 & 26 & Male & Ring fingertip & Excision with V-Y flap repair & No recurrence at 12 months \\
Soto et al. [9] & 2014 & 59 & Female & Left great toe & Excision & No recurrence at 12 months \\
Current report & 2020 & 24 & Male & Left third finger & Shave biopsy and extraction & $\begin{array}{l}\text { No recurrence thus far } \\
\text { (at 6 weeks) }\end{array}$ \\
& & & & & & \\
\hline
\end{tabular}

Table 2. Summary of nail unit schwannoma reports

\begin{tabular}{ll}
\hline Age (mean \pm SD), years & $39 \pm 15$ \\
\hline Most common digits affected & Thumb, ring finger, third finger, and great toe \\
\hline Most common surgical techniques & $\begin{array}{l}\text { Excision in all reports except for shave biopsy } \\
\text { in our report }\end{array}$ \\
\hline Recurrence pattern & No recurrence reported \\
\hline
\end{tabular}

the patient had no recurrence at 12 months. The fourth case was reported in 2014 by Soto et al. [9] in a 59-yearold woman who presented with a subungual schwannoma of the left great toe. The authors used color Doppler ultrasound imaging in addition to histologic findings to illustrate a schwannoma in the nail bed of the left great toe [9].

Interestingly, sonography is emerging as a method to diagnose schwannomas prior to resection. Yaaaoka et al. [10] analyzed the sonographic appearance of several sub- cutaneous nodules, including dermatofibroma, schwannoma, small-sized venous malformations, glomus tumor, and nodular fasciitis. After analyzing 44 subcutaneous nodules, they found that sonography was most useful for diagnosing schwannoma, with 6 of 8 schwannomas diagnosed correctly from sonographic appearance. Schwannomas showed increased posterior echo and Doppler flow signals unlike other tumors [10]. In another study, Lee and Yoon [11] studied the ultrasonographic features of schwannoma of the hand by analyzing the ultrasounds 
of 8 patients who were found to have schwannomas by tissue biopsy. They found that in all cases, schwannomas appeared as cystic lesions with clear margins, while 2 of them had acoustic enhancement without internal flow.

After compiling and analyzing all existing reports, with the inclusion of the current case (Table 2), we found that the average age of patients with nail unit schwannoma is 39 years, affecting the thumb, ring finger, third finger, and great toe. All lesions were sampled with an excisional biopsy, except in our case where a shave biopsy and extraction was employed. None of the reports described recurrence of the nail unit schwannoma. In our patient, no recurrence has been reported at 6 weeks of follow-up thus far.

Based on these few reports in the literature, it is important to include schwannoma in the clinical differential diagnosis of nail unit soft tissue tumors. In addition to schwannoma, the differential diagnosis for nail unit soft tissue tumors could also include neurofibroma, glomus tumor, lipoma, angiolipoma, superficial acral fibromyxoma, dermatofibrosarcoma protuberans, cysts (such as ganglion or myxoid cysts), keratinocyte neoplasms, melanocytic lesions, and nail unit-specific tumors (such as onychomatricoma) as well as others.

\section{Statement of Ethics}

The patient's consent for publication was obtained.

\section{Conflict of Interest Statement}

The authors have no conflicts of interest to disclose.

\section{Funding Sources}

The authors did not receive any funding.

\section{Author Contributions}

M.D. wrote the manuscript and prepared figures, A.F. prepared figures and edited the manuscript, S.P. prepared figures and edited the manuscript, T.O. provided patient care and edited the manuscript, R.E. edited the manuscript, and A.R. supervised writing the manuscript, prepared figures, edited the manuscript, and is the primary pathologist for the case.

\section{References}

1 Kurtkaya-Yapicier Ö, Scheithauer B, Woodruff JM. The pathobiologic spectrum of schwannomas. Histol Histopathol. 2003;18: 925-34.

2 Requena L, Sangueza OP. Benign neoplasms with neural differentiation: a review. Am J Dermatopathol. 1995; 17:75-96.

3 Plotkin SR, Wick A. Neurofibromatosis and schwannomatosis. Semin Neurol. 2018;38: 73-85.

4 Biegel JA, Busse TM, Weissman BE. SWI/SNF chromatin remodeling complexes and cancer. Am J Med Genet Part C Semin Med Genet. 2014;166:350-66.
5 Vindhyal MR, Elshimy G, Elhomsy G. Carney complex. Treasure Island, FL: StatPearls Publishing; 2020.

6 Kulkarni J, Moholkar A, Patil A. Subungual schwannoma: an uncommon location. J Hand Surg Am. 2013;38:1258-9.

7 Moon SE, Cho YJ, Kwon OS. Subungual schwannoma: a rare location. Dermatologic Surg. 2006;31:592-4.

8 Huntley JS, Davie RM, Hooper G. A subungual schwannoma. Plast Reconstr Surg. 2006; 117:712-3.
9 Soto R, Aldunce MJ, Wortsman X, Sazunic I. Subungual schwannoma with clinical, sonographic, and histologic correlation. J Am Podiatr Med Assoc. 2014;104:302-4.

10 Yaaaoka M, Kuramochi A, Kutani K, Katoh $\mathrm{K}$, Saitoh T, Ikebuchi K. Benign subcutaneous nodules for the diagnosis of which ultrasonography is useful. Rinsho Byori. 2016;64: 1229-35

11 Lee SJ, Yoon ST. Ultrasonographic and clinical characteristics of schwannoma of the hand. Clin Orthop Surg. 2017 Mar;9(1):91-5. 